\title{
Second Occurrence Focus and Relativized Stress F
}

\author{
Mats Rooth \\ Linguistics and CIS, Cornell University
}

\section{Semantic and phonological scope of focus}

In "anaphoric" or "givenness" theories of the semantics and pragmatics of intonational focus, the first sentence in (1) is in a certain sense the antecedent for the focus in the second sentence. The representation (2) makes the anaphora explicit using an operator " " which marks the scope and the antecedent of the focus. The focused phrase is marked with the feature $\mathrm{F}$.

(1) You boil your vegetables?

I MICROwave my vegetables.

(2) [You boil your vegetables $]_{8}$

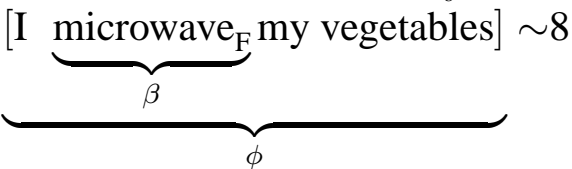

The semantic part of anaphoric theories jointly constrains the denotation of the antecedent (here the proposition 'John boils John's vegetables', assuming the second speaker is John) and the focus semantic value of the scope of the focus (here the set of propositions of the form 'John $R$ 's John's vegetables', where $R$ is an alternative to 'boil'). A couple of versions of the constraint have been given (Rooth, 1992, 1996a; Schwarzschild, 1999). Two options are stated in (3), restricting attention to the clauses which apply to (2). ${ }^{1}$ In order for the structure to be licensed, the focus constraint has to be satisfied. In this case, the first one is satisfied because the antecedent 'John boils John's vegetables' is an element of the focus semantic value of the scope of the focus. The second version is satisfied because the union of the focus semantic value is a disjunction of propositions, and in this case one of them is the antecedent 'John boils John's vegetables'. Since a proposition $p_{i}$ entails the

\footnotetext{
${ }^{1}$ The constraints include several subclauses or type accommodation principles which are conditioned by semantic type.
} 
disjunction $p_{1} \vee \ldots \vee p_{i} \vee \ldots$, the antecedent entails the union of the focus semantic value.

(3) a. Where $\phi$ is the scope of the focus, the denotation of the antecedent is an element of $\llbracket \phi \rrbracket^{\mathrm{f}} . \quad$ (Rooth, 1992)

b. Where $\phi$ is the scope of the focus, the denotation of the antecedent entails the union of $\llbracket \phi \rrbracket^{\mathrm{f}} . \quad$ (Schwarzschild, 1999)

In combination, the notation (2) and its interpretation (3) define a semantic notion of the scope of a focus. ${ }^{2}$ Truckenbrodt (1995) argued that this agrees with a phonological notion of the scope of focus, namely the domain of prominence of the focus. To capture the correlation, he proposed a Stress F constraint which in my discussion will be formulated as (4). ${ }^{3}$

\section{(4) Stress F}

Let $\beta$ be an F-marked phrase with scope $\phi$. Then the strongest stress in the phonological realization of $\phi$ falls within the realization of $\beta$.

Suppose stress is represented in the metrical grid formalism (Prince, 1983), and that the grid representation of (2) is along the lines of (5). Then Stress F is satisfied, because the strongest stress in the phonology of the whole sentence, namely the stress on the first syllable of micro, falls within the phonological interval corresponding to the F-marked element microwave. Stress F would not be satified if the first syllable of vegetables had greater grid prominence than the first syllable of micro.

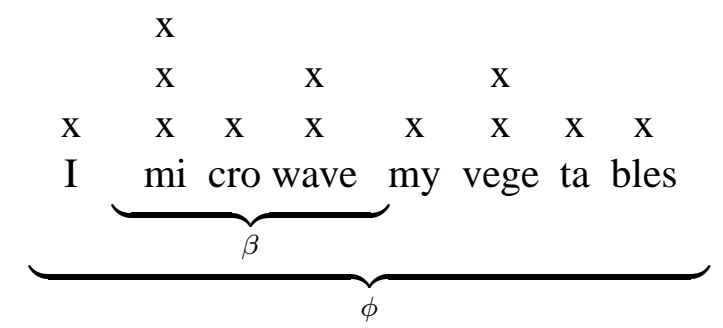

Truckenbrodt's argument for Stress F is based on examples like (6), where there is arguably a focus on American whose scope is the nominal [an American farmer], rather than the whole sentence.

$$
\left[_{\mathrm{S}}\left[_{\mathrm{NP}} \text { an } \text { American }_{\mathrm{F}} \text { farmer }\right]_{1} \sim 2 \text { told a }\left[\left[_{\mathrm{NP}} \text { a Canadian }_{\mathrm{F}} \text { farmer }_{2} \sim 1\right] \text { a joke }\right]\right.
$$

In the anaphoric theory, the scope of the foci on American and Canadian can not be the whole sentence, because the sentence can be used in discourse contexts where there is no available antecedent which has the form 'a $P$ farmer told a $Q$ farmer a joke', or which entails that a farmer of some nationality told a farmer of some

\footnotetext{
${ }^{2}$ In Schwarzschild (1999), The scope of an $\mathrm{F}$ is marked by the next $\mathrm{F}$ up, rather than the $\sim$ operator.

${ }^{3}$ See also Jackendoff (1977), Rooth (1996), Büring (2006), Selkirk (2006).
} 
nationality a joke. In the representation (6) with a narrower scope for the focus on American, the constraint (3a) is satisfied for the focus on American, because as long as being Canadian is an alternative to being American, the denotation of the antecedent [a Canadian farmer] is an element of the focus semantic value for the scope of the focus. ${ }^{4}$

The point now is that the semantically-motivated scope of focus in (6) agrees with the phonological domain of prominence. (7) is a plausible grid representation. The second syllable in American has greater stress than anything else in the phonological interval corresponding to [an American farmer], but it does not have greater stress than joke or the second syllable in Canadian. ${ }^{5}$ But stress F is obeyed, because the semantic scope of the F on American (the $\phi$ in the constraint) is the nominal [an American farmer], rather than the whole sentence.

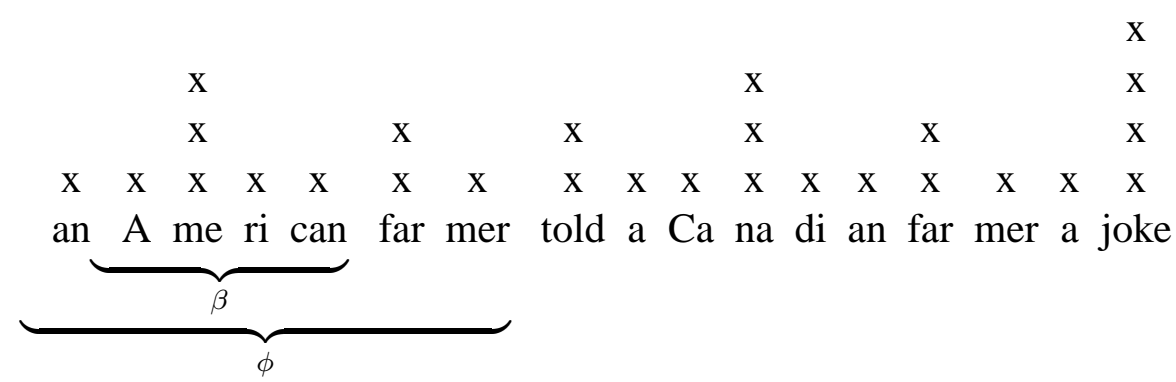

The argument supports the hypothesis that the semantic scope of focus matches phonological domain of focus prominence. This is a substantial empirical and theoretical claim, and one that is attractive because it postulates a kind of homomorphy between phonology and semantics. However, the homomorphy is arguably poorly articulated in (4), because there is reference to information that is scattered in the syntactic tree and in the phonology, and to a correspondence between focused phrases, their scopes, and intervals in a phonological representation. This is explained in Section 2. As a corrective, an architecture is proposed with local operators that have a local phonological interpretation of relative prominence, and a local semantic interpretation in terms of the scope of focus. Section 3 widens the empirical domain to include second occurrence focus configurations, which are complex configurations where two focused phrases take different, nested scopes.

\footnotetext{
${ }^{4}$ The semantic side of the story about the focus on Canadian is symmetric. However, Féry and Samek-Lodovici (2006) point out that there is an asymmetry on the phonological side. In their example (i), each occurrence of Cheverolet can be within the scope of the focus on the occurrence of farmer to the left. But the first occurrence of Chevrolet is realized with an accent.

(i) An American farmer with a purple Chevrolet was talking to a Canadian farmer with a purple Chevrolet.

${ }^{5}$ Evidence for this is that the nuclear accent falls on joke. Truckenbrodt notes "If the phonological domain of a focus would be the clause, regardless of the semantic domain the clause-final defaultstress in these examples would not be derived. Instead, one of the foci in each of these examples would attract the nuclear stress of the clause."
} 


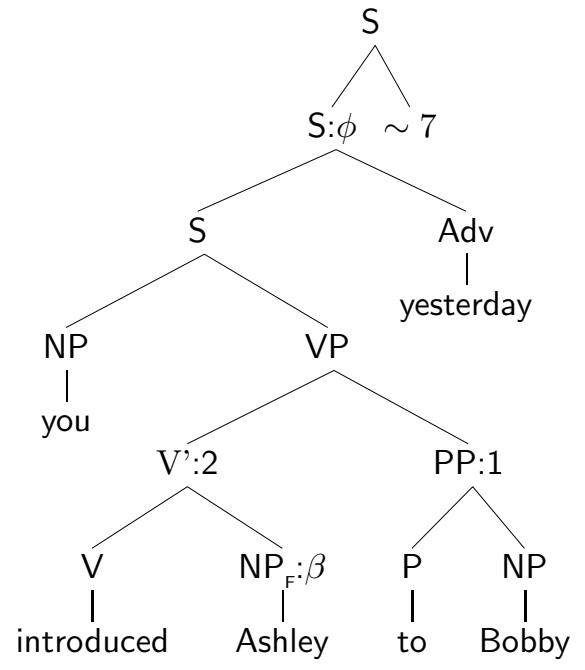

Figure 1: An application of Stress F. $\beta$ is the focus and $\phi$ is its scope.

The scope relationships in some second occurrence configurations contradict the simple phonology-semantics correspondence in Stress-F. This problem is addressed by a "relativized" statement of Stress-F, which allows a single phrase to contain focused subconstituents that take different scopes outside the phrase. Section 4 takes up the technical problem of providing a semantics for the local operators proposed in Section 2, and showing how the complex configurations discussed in Section 3 are analyzed. Section 5 sums up.

\section{The local operator architecture}

Figure 1 illustrates a structure of application for Stress-F. In an embedded position, there is an F-marked phrase Ashley, which is the $\beta$ of (4). Its scope is the entire clause, which is the $\phi$ of (4). Applying Strees-F requires (i) locating the phonological interval $\phi^{\prime}$ that corresponds to $\phi$, (i) locating the phonological interval $\beta^{\prime}$ that corresponds to $\beta$, and (iii) checking whether the syllable $\sigma$ in $\phi^{\prime}$ with maximal prominence in $\phi^{\prime}$ falls within $\beta^{\prime}$. This procedure looks at two levels of the syntactic tree that are separated from each other by three nodes, and it refers at two levels to a correspondence between syntactic phrases and phonological intervals. Although a statement of the syntax-phonology interface defines a map between syntax and phonology, determining that a phrase $\phi$ has phonological realization $\phi^{\prime}$, it is a different matter for a grammatical constraint to refer to multiple pairs that stand in the realization relation, as stress $\mathrm{F}$ does when it refers to the syntax-phonology pairs $\left\langle\phi, \phi^{\prime}\right\rangle$ and $\left\langle\beta, \beta^{\prime}\right\rangle$. In these respects Stress-F, rather than stating an elegant homomorphy between syntax and phonology, is an implausibly complex global constraint. Section 3 will show that this problem becomes even more severe in config- 


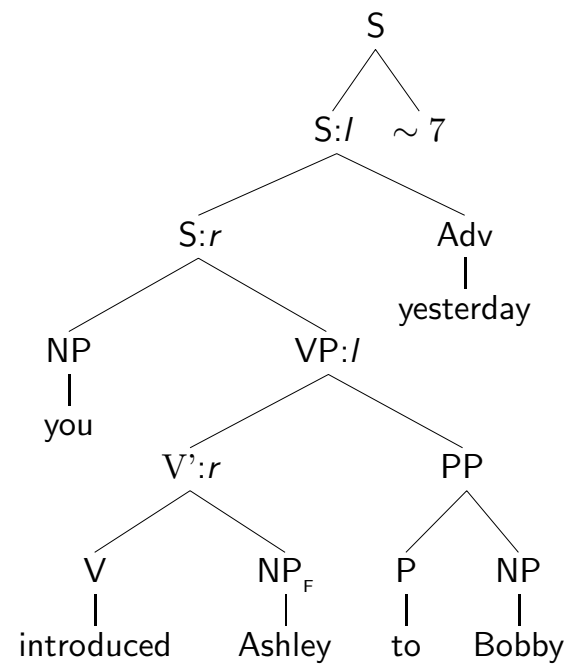

Figure 2: The scope of focus represented with local prominence operators.

urations involving several F's with different scopes. But even in basic applications like the one in Figure 1, Stress $F$ has the character of a descriptive generalization which is not a plausible candidate for a constraint linking linguistic levels, because it refers to so many things at once.

The alternative is to state a local correlation between phonological and semantic interpretation. Rather than stating a global constraint referring to the syntaxphonology correspondence at two tree levels, one would like to state a local correspondence at each tree level. The phonological side of this correspondence in the approach developed here is the familiar one of relative metrical prominence in a binary-branching tree. Phonologically, marking the scope of a focus involves local demarkations of the relative prominence of the two children of binary-branching nodes. In a binary branching configuration $[\gamma \psi]$, the scope of a focus in $\gamma$ is expanded only if $\gamma$ is phonologically more prominent than $\psi$. This is reminiscent of the $s w$ architecture for metrical trees proposed in Liberman and Prince (1977), where a binary branching node $[\gamma \psi]$ where $\gamma$ is more prominent is represented as $\left[\gamma_{\mathrm{s}} \psi_{\mathrm{w}}\right]$. For reasons related to the semantics, this prominence relation is represented here as $[\gamma \psi]_{1}$, with 1 indicating prominence on the left. Prominence on the right is indicated with $[\gamma \psi]_{\mathrm{r}}$.

This results in the revised representation in Figure 2 for the example where Stress F was applied in Figure 1. On the phonological side, it should be apparent how prominence relations have been localized. Instead of referring globally to the phonological domain of prominence $\phi^{\prime}$ of the prominence in $\beta^{\prime}$, we have local operators 1 and $r$ which represent that $\beta^{\prime}$ is maximally prominent withing $\phi^{\prime}$. Semantically, the operator 1 should expand the scope of a focus in the left child of the node that bears it, while $r$ expands the scope of a focus from the right child. The effect should be that the scope of the focus on Ashley in Figure 2 is expanded to the 
clausal level. This semantic side of the architecture is worked out in Section 4.

\section{Stress $\mathbf{F}$ in second occurrence focus configurations}

Second occurrence focus is an intonational pattern found in certain examples with multiple motivations for focus: ${ }^{6}$ )

(8) a. Eva only gave xerox copies to the GRADUATE F $_{\mathrm{F}}$ students.

No, PETR $_{\mathrm{F}}$ only gave xerox copies to the graduate ${ }_{\mathrm{SOF}}$ students.

b. We only introduced Marilyn to $\mathrm{JOHN}_{\mathrm{F}}$ Kennedy.

(i.e. not to Bobby and Edward Kennedy)

We also only introduced $\mathrm{SUE}_{\mathrm{F}}$ to $\mathrm{John}_{\mathrm{SOF}}$ Kennedy.

c. Mary only STEAMS vegetables, and even $\mathrm{JOHN}_{\mathrm{F}}$ only steams $\mathrm{SOF}_{\text {vegeta- }}$ bles.

The notation SOF marks a phrase where on semantic grounds one might expect there to be a focus, but where apparently there is no focus marked by a pitch movement. In early literature, there was disagreement about phonetic/phonological status of SOF. Either SOF is not phonetically marked at all (Partee, 1991; Krifka, 2004) or SOF is phonologically prominent, though not marked with a pitch accent (Rooth, 1992; von Fintel, 1994; Rooth, 1996b). Recent experimental studies are interpreted as supporting the second position, though the magnitide of the phonetic reflexes is small and not consistent at the token level (Beaver et al., 2007; Howell, 2007). There is additional evidence from weak pronouns (von Fintel, 1994; Rooth, 1996b; Beaver et al., 2007). English pronouns can be reduced to various degrees; at the extreme, they can lose their onset and be prosodically incorporated into the preceding word as in (9). This process is blocked in SOF configurations, as illustrated in (10).

(9) a. I likim (= him).

b. He likser $(=$ her $)$.

(10) Mary's boyfriend only likes HER. \#Even her BOSS only likser.

The SOF data are interpreted as indicating that the phonological correlate of $\mathrm{F}$ is metrical prominence, as in Stress F theories, rather than pitch accent (Rooth, 1996b; Selkirk, 2006a; Beaver et al., 2007). Some prominences derived from F surface with pitch accents; these are the ordinary F's. Others do not; these are the SOF's. Apparently, all examples of SOF in the literature have a special configuration of relative scope of focus, with the scope of the SOF embedded in the scope of the primary $\mathrm{F}$ (Rooth, 1996b; Büring, 2006; Selkirk, 2006a). The configuration is schematized in (11).

\footnotetext{
${ }^{6}$ These examples are from Partee (1991), Rooth (1993), and Krifka (2004).
} 


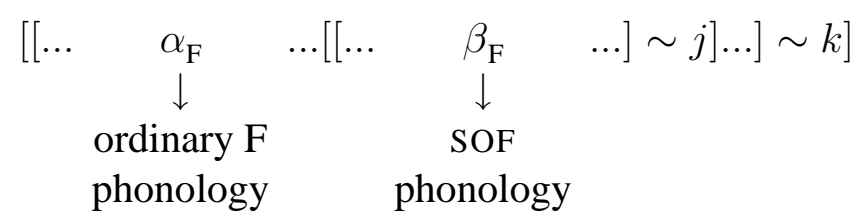

An additional line of evidence is provided in Féry and Ishihara (2005). They look at examples in German where the narrower-scope focus precedes the widerscope focus. They find that in such configurations the narrower-scope focus is realized with a pitch accent, but one which has a compressed pitch range relative to the wider-scope focus.

We are left with the following picture. In a configuration with two F's whose scopes are nested, the narrower scope focus is realized with less phonological prominence than the wide scope focus. This provides the interface between semantics and phonology/phonetics. The prominence relation results in different realizations, depending on linear order. The narrower-scope focus, if it follows the wider-scope one, is realized with SOF phonology and phonetics, without a pitch movement. If the narrower-scope focus precedes the wider-scope one, it is realized with a compressed pitch range.

The phonology-semantic correlation seen in second occurrence focus-with the wider scope focus being realized with greater prominence-is reminiscent of the phonology-semantics homomorphy expressed by Stress F. Are the prominence relations seen in SOF in fact a consequence of Stress-F, or of the reformulation of Stress F in terms of local operators? Consider the nested-focus example (12), where in the second sentence the focus on Ashley is realized with SOF phonology.

(12) John said you only introduced Ashley ${ }_{F}$ to Bobby yesterday. Wrong. Mary ${ }_{F}$ said you only introduced Ashley $_{F}$ to Bobby yesterday.

The embedded clause in the second sentence of (12) should have a representation similar to Figure 2, with a focus on Ashley taking scope at the level of the embedded clause. The focus on the matrix subject $F$ has scope at the matrix S. Using local prominence operators to represent the scopes, this results in Figure 3 . The operator $l$ at the top has the semantic function of extending the scope of the focus on Mary to the matrix. At the same time, it has a phonological interpretation of relative prominence, indicating that the matrix subject is more prominent than the matrix VP. Since the focus on Ashley is embedded in the matrix VP, it follows that the the matrix subject, with the wide scope focus, is more prominent than the embedded object Ashley, with the narrow-scope focus. In other words, the prominence relations seen in SOF fall out of the semantically motivated representation of scope of focus, once scope of focus is represented by local operators with both semantic and phonological interpretations.

The same result is obtained with Stress F in the formulation 4. Starting with the more embedded focus, [Ashley $]_{\mathrm{F}}$ is $\beta_{1}$ and its scope $\phi_{1}$ is the embedded clause. Stress $\mathrm{F}$ requires that the most prominent syllable in the phonological interval $\phi_{1}{ }^{\prime}$, 


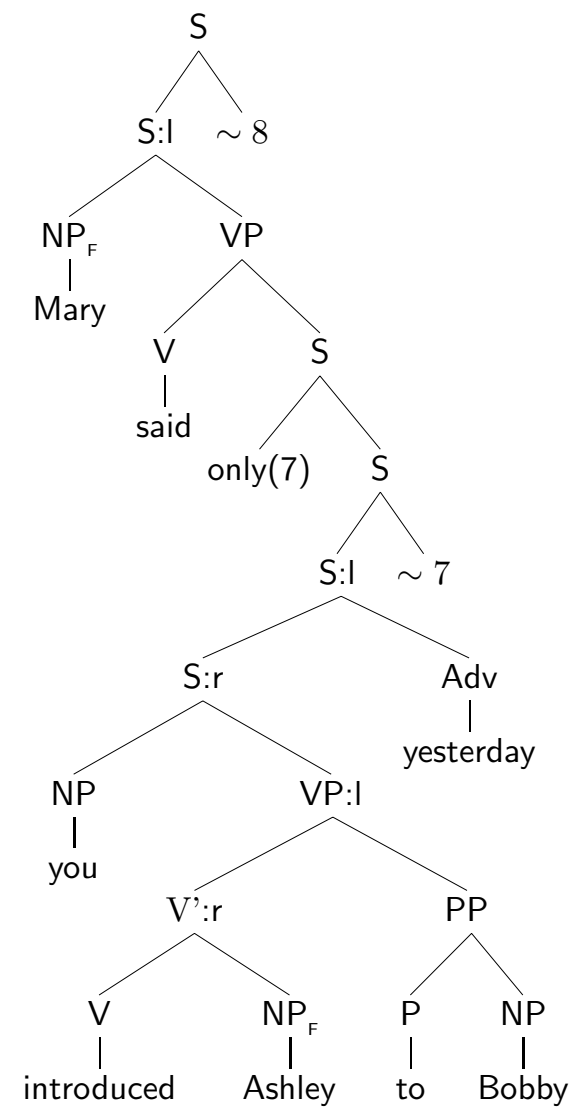

Figure 3: Local prominence operators in an SOF configuration.

the phonological realization of the embedded clause, falls within $\beta_{1}{ }^{\prime}$, the phonological realization of the focused phrase Ashley. Looking at the higher focus, Stress $F$ requires that the most prominent syllable withing the realization $\phi_{2}{ }^{\prime}$ of the entire sentence fall within the realization $\beta_{2}{ }^{\prime}$ of the focused phrase Mary. Given the subinterval relations in (13), it follows that the most prominent syllable in Mary is more prominent than the most prominent syllable in Ashley. This is as desired.

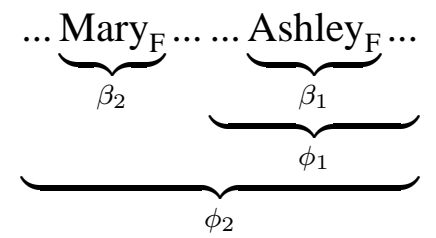

The logic in this form unfortunately falls apart in SOF examples where there is a single phrase $\psi$ that dominates both F's, and is dominated by the scopes of both F's. Consider example (14), where there is an F on Ashley with ordinary realization, and and F on Bobby with SOF realization. Example (15) is similar, with F on Gouda and caviar, and SOF on New Jersey. 


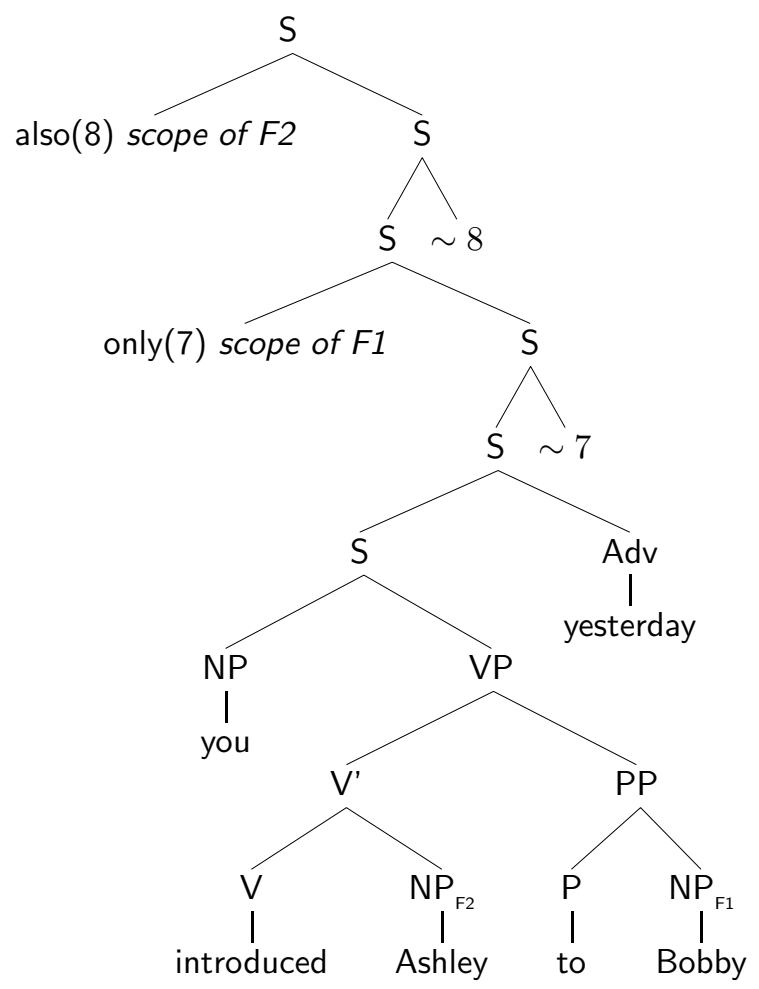

Figure 4: Scopes of F in example (14). F2 informally annotates the wider scope focus, and F1 the narrower scope focus.

(14) You know what? You only introduced Mona to Bobby ${ }_{\mathrm{F}}$ yesterday.

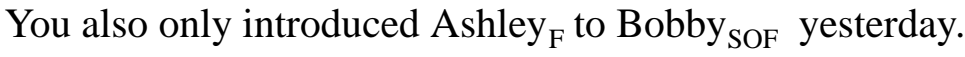

(15) What foods did you only find in New Jersey last year?

I only found Gouda and Caviar ${ }_{\mathrm{F}}$ in New Jersey SOF $_{\text {last year. }}$

Figure 4 represents the scopes of focus features in (14). The indexing on F is informal notation for the scope of focus: F1 takes scope at the first c-commanding $\sim$, while F2 takes scope at the second c-commanding $\sim$. The hope is that when the scope relations are expressed with local $l r$ operators, the phonological promimence relations will fall out. Instead, there is a contradiction. The tree on the left in Figure 5 is decorated with the operators in the embedded clause that represent the scope of F1. The tree in the right has the operators that represent the scope of F2. The contradiction is seen at the VP node, which is labeled $\mathrm{r}$ in the tree representing the scope of F1, and 1 in the tree representing the scope of F2.

Another way of seeing the problem is to apply Stress F as formulated in (4) to the tree in Figure 4. Starting with F1, Bobby is the focused element $\beta_{1}$, and [you introduced Ashley to Bobby] is the scope $\phi_{1}$ of the focus. Stress F tells us that the highest stress in $\phi_{1}$ falls within $\beta_{1}$. Second, let's apply Stress-F to F2, with Ashley 


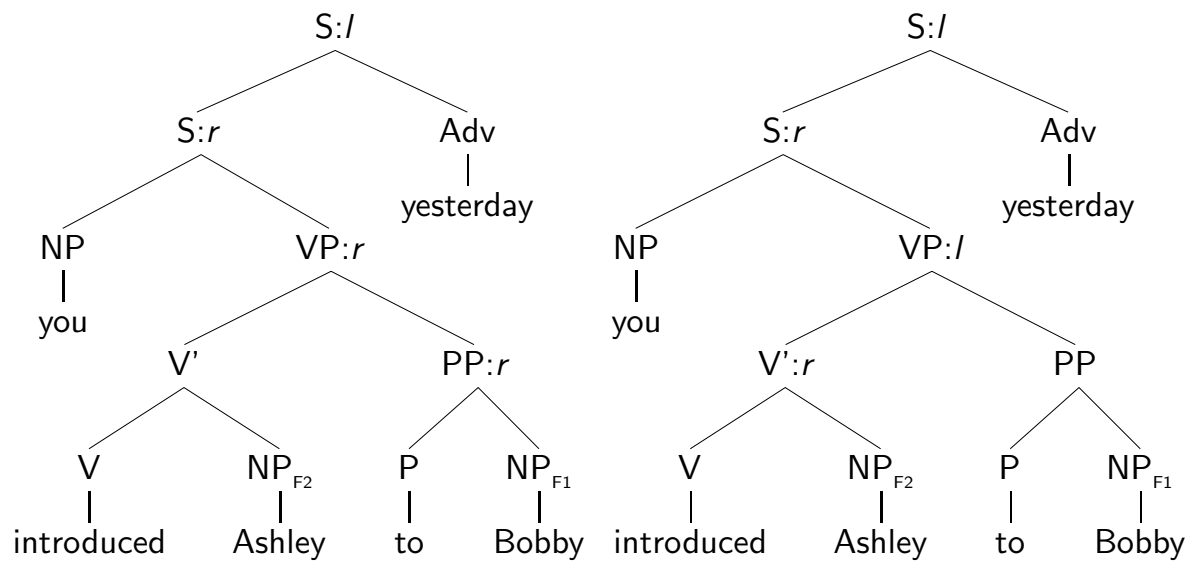

Figure 5: On the left, local operators in the embedded clause expressing the scope of F1. On the right, local operators expressing the scope of F2.

as the focused element $\beta_{2}$, and [you only introduced Ashley to Bobby] as the scope $\phi_{2}$ of the focus. Stress-F tells us that the highest strees within $\phi_{2}$ must fall within $\beta_{2}$. These constraints are inconsistent, because $\beta_{1}$ and $\beta_{2}$ are disjoint subintervals of $\phi_{1}$, while $\phi_{1}$ is a subinterval of $\phi_{2}$. Under these circumstances, $\beta_{1}$ can not contain the greatest prominence in $\phi_{1}$ while $\beta_{2}$ contains the greatest prominence in $\phi_{2}$. This would be comparable to the tallest mountain in North America being in Alaska, while the tallest mountain in the Americas is in Colorado. This logic was discovered by Daniel Büring (Büring, 2006).

To deal with the problem in a rule similar to Truckenbrodt's Stress-F, one has to "relativize" the constraint to allow for two F's to take different scopes out of the same phrase. This can be done by stipulating that wider scope F's should be ignored in checking Stress F. This results in (16).

\section{Relativized Stress-F}

Let $\beta$ be an F-marked phrase with scope $\phi$. Then the strongest stress in the phonological interval corresponding to $\beta$ is strictly stronger than any stress in the phonological interval corresponding to $\phi$ which is not contained in the phonological interval corresponding to an F-marked subconstituent of $\phi$ whose scope is at least $\phi$.

In Figure 4, Relativized Stress-F as applied to F1 says that we should ignore prominences within $\beta_{2}$ (which is [Ashley $]_{\mathrm{F} 2}$ ) while checking whether the greatest prominence within $\phi_{1}$ falls within $\beta_{1}$ (which is [ Bobby $]_{\mathrm{F} 1}$ ). Suppose the grid structure for $\phi_{1}$ is along the lines of (17). The first syllable of Ashley has the greatest prominence. But in checking relativized Strees $\mathrm{F}$ for the focus on Bobby, any prominences within Ashley are ignored, because Ashley is contained in a wider-scope focus. Relativized Strees F applied to F2 on Ashley requires that the the first syllable on Ashley have greater prominence than the first syllable on Bobby, which it does 
in this representation. So the contradiction is removed. In general, relativization allows two F's to take different scopes out of the same constituent, something which is not possible under Stress F. At the same time, it entails that the wider scope focus have greater stress prominence.

(17) .

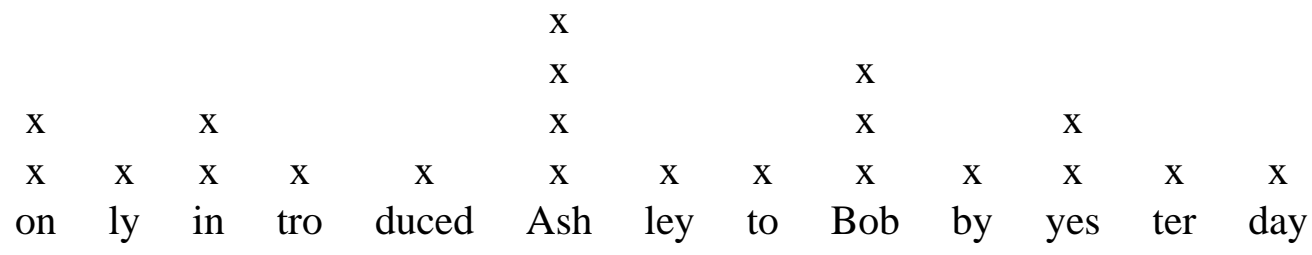

If as maintained in Section 2 the original Stress F is a complicated global statement that refers to too many pieces of representation at once, then Relativized Stress-F is much worse-it obviously has the status of a descriptive generalization which should be derived from simpler primitives. This is the project of Section 4 . Before proceeding with this, I would like to back up to reconsider the empirical status of examples (14) and (15). Schwarzschild (2004) and Büring (2006) discussed examples like (18) that in the scope of F's are isomorphic to (14) and (15). According to them, there is no way of pronouncing (18b) which fits in with the question context (18a). On these intuitions, neither placing the nuclear accent on crepes (18c), or on Paris (18d) works.

(18)a. What food will Renee only eat in Paris?

b. She'll only eat crepes in Paris.

c. \# She'll only eat CREpes in Paris.

d. \#She'll only eat crepes in PARis.

I think it is not advisable to draw any deep conclusions from (18), because when it is adjusted by adding some material after the last focus as in (19), by adding some syllables after the stressed syllable marking the first focus as in (20,) or both (21), it becomes good. Presumably, they help because they add non-prominent material relative to which the SOF can be perceived as prominent. These adjustments do not affect the scope of focus. If (19)-(21) are good, they are counterexamples to the simple Stress F rule, and are problematic for the local operator architecture in the way explained in connection with Figures 4 and 5.

(19) What foods will Renee only eat in Paris next year?

She'll only eat CREPES in Paris next near.

(20) What foods will Paul only eat in Paris?

He'll only UDO noodles in Paris.

(21) What foods will Renee only eat in Paris next year?

She'll only eat UDO noodles in Paris next near.

But what about (18)? Maybe some speakers percieve it as a garden path, where 
because there is no phonetic evidence for a focus on Paris, the only reading which is recovered is one where only is associated with a focus on crepes. Another way of improving (18c) is to put a rising intonation indicating a partial answer on the first focus crepes. With this intonation and pragmatics, the example seems perfect. The rising intonation perhaps removes the garden path effect, because the focus with a rising intonation can not in this example be read as associated with only.

A slightly different hypothesis is that a purely phonological constraint on good metrical configurations is responsible for the judgment that (18) is bad. As pointed out to me by Roger Higgins, there is an unpronouncability effect in (22). Again, the example is saved by adding unstressed syllables after the stressed syllable marking the first focus as in (23). The relation between (22) and (23) is similar to the one between (18) and (20).

(22) Who does only John like?

??Only $\mathrm{John}_{\mathrm{F}}$ likes Mary ${ }_{\mathrm{F}}$.

(23) Who does only Abernathy like?

Only Abernathy $_{F} / /$ likes Mary .

Of course, if the data are different, the theory should be different. Büring (2006) has it that SOF examples with the scope pattern in like the one in (18) are bad, and attributes this to the syntax-phonology interface in the form of ordinary Stress-F. The logic is that in a represention like Figure 4, unrelativized stress F can not be satified, as reviewed above.

Tentatively, I will assume that scope configuration in Figure 4 is possible. Hedging this, though, any claims about English prosody needs to be tested in a laboratory setting and documented in corpus data. As far as I know, no laboratory work on SOF on bears on the configuration in Figure 4.

\section{Local operators generalized}

Here is an excercise in labeling trees. Starting with the tree in Figure 4, mechanically percolate the F-indices 1 and 2 to the level of their scopes. The indices are written in descending order at each node. This produces the tree on the left in Figure 6. Next, mechanically add an additional annotation which indicates whether each index came from the left or the right in the binary tree. In the sequence 2,1 which labels [ ${ }_{\mathrm{VP}}$ indroduced Ashley to Bobby], the index 2 came the left child (from [introduced Ashley]), while the 1 came from the right child (from [to Bobby]). This results in the label " $l r$ ", with " $l$ " indicating a source on the left, " $r$ " indicating a source on the right, and the linear order in " $l r$ " corresponding to the linear order in " 2,1 ". In the right tree in Figure 6, all nodes are labeled according to this principle. It can be observed that the $l$ 's and $r$ 's capture all the information about the scope of the F's, so that if we include $l$ and $r$ in the representation, the F indices (here 1 and 2) are no longer required. 


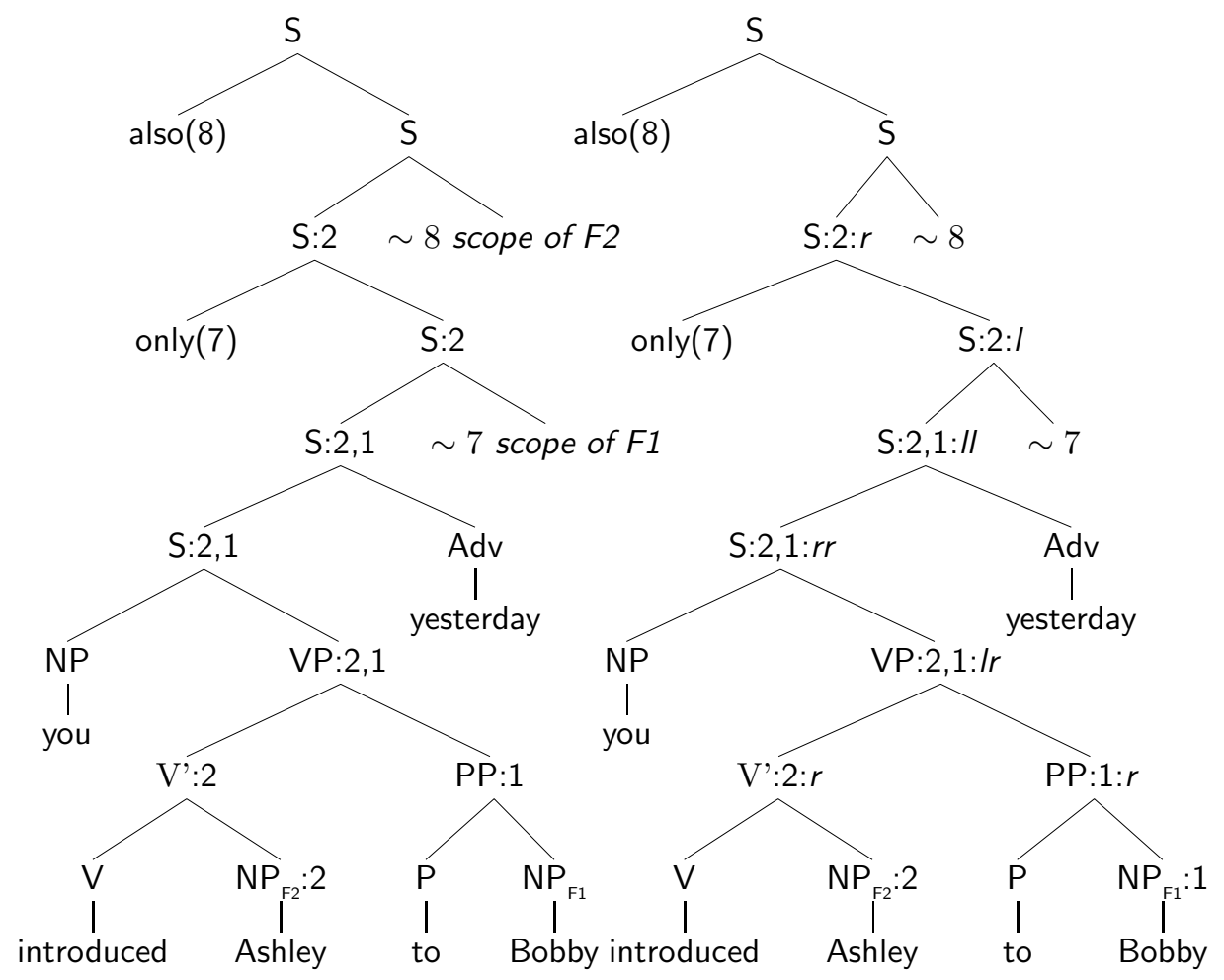

Figure 6: $l r$ annotations added in Figure 4.

This procedure represents the scope of focus using a sequence of $l r$ operators at each node, rather than with single operators as in Section 2. This allows the scope of multiple F's to be represented. As before, $l$ and $r$ are operators which have both a phonological interpretation and a semantic one. ${ }^{7}$ Semantically, a sequence $l r$ indicates that there is a widest-scope focus in the left child, and an additional focus in the right child. A sequence $r r$ indicates that there is a widest-scope $\mathrm{F}$ in the right child, and an additional $\mathrm{F}$ also in the right child. And so forth. Turning to phonology, the phonological interpretation of any sequence $l \alpha$ which begins with an $l$ is that the left child of the labeled node must be metrically stronger than the right child. The phonological interpretation of a sequence $r \alpha$ which begins with $r$ is that the right child must be stronger than the left one. So in a sequence with more than one operator, only the first one counts phonologically. This resolves the contradiction in Figure 5. The VP node has the lable $l r$, and so the left daughter of the VP, which embeds the primary focus, is more prominent.

My starting point for defining the semantic part of the local-operator architecture is the compositional structured-meaning analysis of Krifka (1991). ${ }^{8}$ As in all

\footnotetext{
${ }^{7}$ This is somehow similar to Wagner (2006)'s local alternative semantics, which uses a local operator on a pair of sisters pair of sisters which has a semantic and a phonological interpretation.

${ }^{8}$ I don't contemplate using structured meanings as a semantics for focus in place of
} 
structured meaning approaches to focus, the idea is that focus has the effect of structuring the semantic value into a tuple von Stechow (8589). ${ }^{9}$ The denotation of (24a) is normally (24b). ${ }^{10}$ It is structured into the tuple (24c). The first element is a version of the normal denotation with a bound variable in the position of the focused phrase, and the second element is the denotation of the focused phrase. I will call the first elements in structured meanings "focus skeletons". For another example, the structured interpretation of the focused phrase (24d) is (24e), where the skeleton is the identity function. As in Krifka's compositional approach, structuring happens also at compositional levels, not just at the level of the scope of the focus.

(24)a. [ [ indroduce Ashley ${ }_{\mathrm{F}}$ ]

b. (introduce ashley)

c. $\langle\lambda z($ introduce $z)$, ashley $\rangle$

d. Ashley $_{\mathrm{F}}$

e. $\langle\lambda y . y$, Ashley $\rangle$

On the approach to be developed here, a phrase containing several unbound foci denotes a tuple whose first element is a focus skeleton with the focus positions bound by lambda, whose second element is the denotion of the widest-scope focus, whose third element is the denotation of the next-widest scope focus, and so forth. $l$ and $r$ are construed as operators which manipulate structured meanings of this kind, determining the scope of F's, while also having their phonological effect. Consider the following scheme. All syntactic branching is binary. Each non-terminal node is annotated with a type-raised version of the ordinary semantic operation for the node, which is usually function application (either in the right or left direction). (25) is the semantic derivation tree for [introduced Ashley] with no focus. At terminal nodes, a type-raising operator $\mathbf{u}$ introduces a unit list, to initialize structured meanings. The operator $\mathbf{a}$ is a type-raised version of the rightward function application operator $\lambda f \lambda a . f a$, and $c$ is an additional operator involved in propagating structured meanings. To obtain the interpretation of the phrase, the lambda term annotating the parent is applied to the lambda terms coming from the two children, in their linear order. In this example, the propagation of structured meanings in the resulting term ca (u introduce) ( $\mathbf{u}$ Ashley) is trivial, producing the unit set of the ordinary semantic value (introduceAshley).

anaphoric/givenness semantics, just as a compositional device.

${ }^{9}$ An option closer to Wold (1996) would be to use a pair of denotations, one of which is a focus skeleton with lambda-bound variables in the position of focused phrases. As in alternative semantics, in a phrase with one focus the other denotation is the ordinary semantic value. In the focus skeleton, the argument order corresponds to the scope of focus. I believe the technical development would be similar to the one in the text.

${ }^{10}$ In this notation a function application term is written $f a$ or $(f a)$ rather than $f(a)$. Application terms are interpreted left-associatively, so that $f x y$ is $((f x) y)$. 


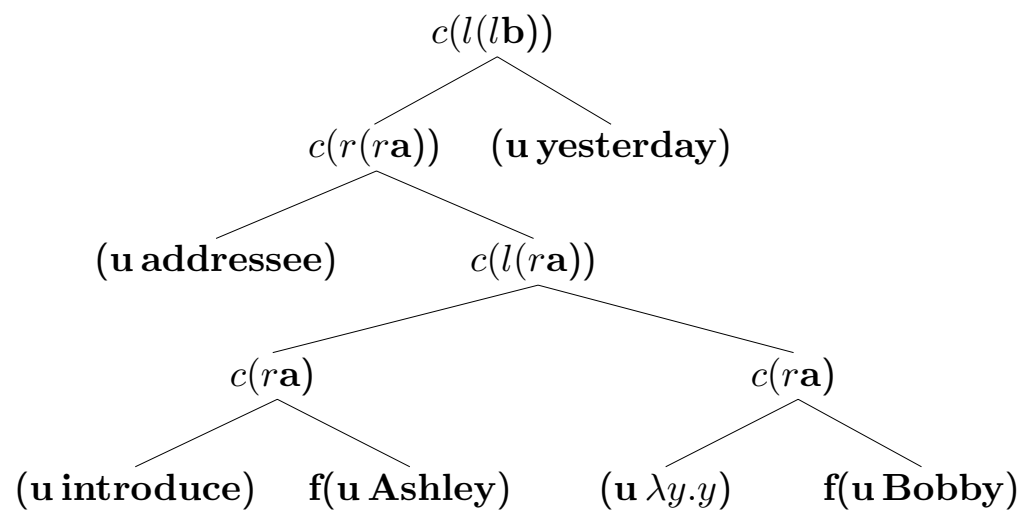

Figure 7: Compositional structure with wider scope for the focus on Ashley

(25)

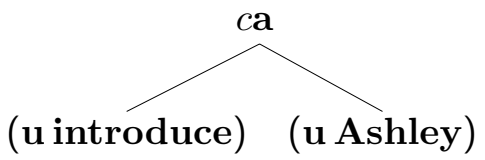

The operators $l$ and $r$ are treated as type-raising operators modifying the semantic operation. They provide for the appropriate projection of structured meanings, in accord with the constraint that $l$ projects a widest-scope focus from the left, while $r$ projects a widest-scope focus from the right. (26) is the compositional structure for (24a), which has a focus on the right. The operator $c(r \mathbf{a})$ which annotatates the parent is a type-raised version of the rightward function application operator which propagates a focus from the right. $\mathbf{f}$ is the semantic focusing operator. The denotation of the whole phrase, which can be written $c(r \mathbf{a})(\mathbf{u}$ introduce $)(\mathbf{f}(\mathbf{u}$ Ashley $))$, is the non-trivial structured meaning $(24 \mathrm{c})$.

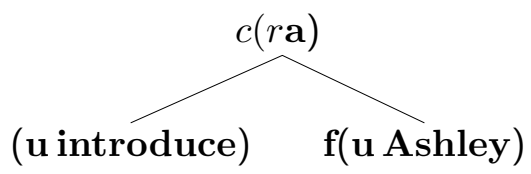

In Figure 7, the scheme is applied to the tree on the right in Figure 6, restricting attention to the part below the focus interpretation operators. The scope of the F's is fixed by the operator $l(r \mathbf{a})$, which propagates the widest-scope focus from the left child [introduce Ashley ${ }_{F}$ ], and the narrower-scope focus from the right child [to Bobby $\left._{\mathrm{F}}\right]$. Figure 8 is the version with the wider-scope focus on $\left[_{\mathrm{NP}}\right.$ Bobby]. Everything is the same, except that $r(l \mathbf{a})$ replaces $l(r \mathbf{a})$ in the semantic operation for the phrase $\left[_{\mathrm{VP}}\right.$ introduce Ashley ${ }_{\mathrm{F}}$ to Bobby ${ }_{\mathrm{F}}$. $r(l \mathbf{a})$ propagates the widest-scope focus from the right child $\left[_{\mathrm{PP}}\right.$ to $\left.\mathrm{Bobby}_{\mathrm{F}}\right]$, and the other focus from the left child [introduced $\left._{\text {Ashley }}\right]$. The difference in focus scope is localized at the point where the denotation of [introduce Ashley ${ }_{F}$ ] combines with the denotion of [to Bobby ${ }_{F}$. Here it has a local phonological effect (on the relative prominence of the two phrases) and a local semantic one (on the scopes of the two F's).

Structured meanings are formalized as lists. To be systematic, even phrases 


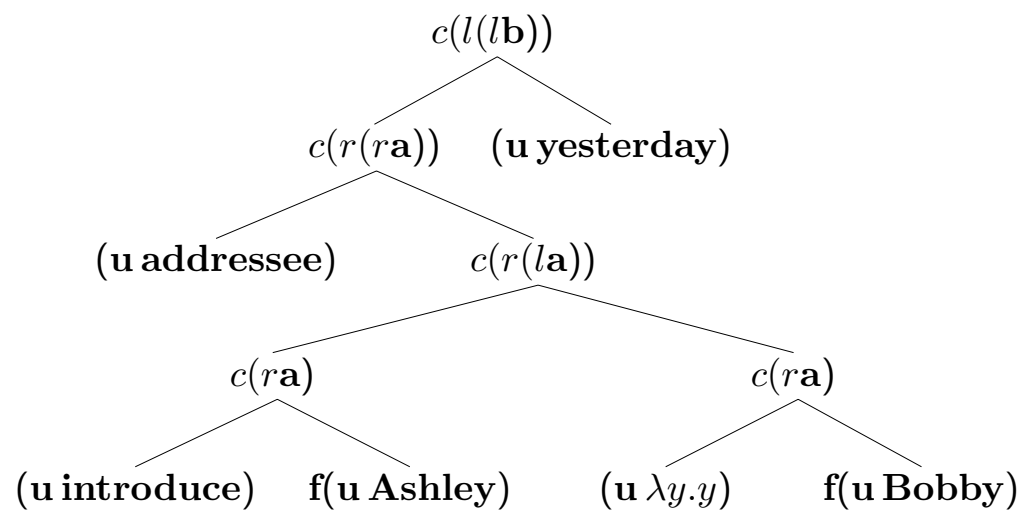

Figure 8: Compositional structure with wider scope for the focus on Bobby

without any F's have list denotations. This illustrated by (25), where each phrase denotes the unit set of its standard semantics. Semantic operations have to manipulate lists, and also, it will turn out, ordered pairs. This is done with the lambda calculus encodings of list and pairing operations stated in Figure 9. ${ }^{11}$ Using these definitions, the list denoted by $\left[_{\mathrm{NP}} \text { Ashley }\right]_{\mathrm{F}}$ is obtained as cns $(\lambda z . z)(\mathbf{u}$ Ashley). Where $L$ is that list, the first element can be extracted as hd $L$, and the second element as hd $(\mathbf{t l} L)$. The non-focused verb introduced denotes a unit list, which can be written as cns introduce nil. To interpret the complex phrase [introduced Ashley $_{F}$ ], the two structured meanings have to be combined. If there was no focus or structuring, the denotations of introduce and Ashley would be combined with the rightward function application operator $\lambda f . \lambda a . f a$. In the structured-meaning scheme, they are instead combined using $\mathrm{cra}$. This is a type-raised rightward function application operator, modified by an operator $r$ which projects a focus from the second (right) argument.

Operators which project structured meanings are defined in two parts. First, there are operators $l_{1}$ and $r_{1}$ which modify the semantic operation which applies to the focus skeletons. Where $o_{1}$ is such an operation, $l_{1}\left(o_{1}\right)$ accommodates an additional focus coming from the left child, and $r_{1}\left(o_{1}\right)$ accommodates an additional focus coming from the right child.

Second, there are operators $l_{2}$ and $r_{2}$ which manipulate the additional meaning components corresponding to focused phrases. Lists of focused-phrase denotations have to be merged together, observing the constraint that $l$ projects the widest-scope focus from the left argument, and $r$ from the right argument. Where $o_{2}$ is an operator which manipulates focus lists, $l_{2}\left(O_{2}\right)$ accommodates an additional focus coming from the left child (corresponding to the first argument of the operator) and $r_{2}\left(o_{2}\right)$ accommodates an additional focus coming from the right child (corresponding to the second argument of the operator.)

\footnotetext{
${ }^{11}$ These definitions are as in Paulson (1996, 386-387). I checked semantic derivations using an implementation in SML of normal-order lambda reduction (Sestoft, 1996).
} 


$$
\begin{array}{ll}
\text { pr } & =\lambda x \cdot \lambda y \cdot \lambda s \cdot s x y \\
\mathbf{f s t} & =\lambda p \cdot p(\lambda x \cdot \lambda y \cdot x) \\
\text { snd } & =\lambda p \cdot p(\lambda x \cdot \lambda y \cdot y) \\
\text { nil } & =\lambda x \cdot x \\
\text { hd } & =\lambda x \cdot \mathbf{f s t}(\mathbf{s n d} x) \\
\text { tl } & =\lambda x \cdot \operatorname{snd}(\mathbf{s n d} x) \\
\text { cns } & =\lambda x \cdot \lambda y \cdot \mathbf{p r}(\lambda x \cdot \lambda y \cdot y)(\mathbf{p r} x y) \\
\mathbf{u} & =\lambda x \cdot \operatorname{cns} x \mathbf{n i l}
\end{array}
$$

( $\operatorname{pr} x y)$ is the ordered pair of $x$ and $y$ fst $p$ is the first element of the ordered pair $p$

snd $p$ is the second element of the ordered pair $p$

empty list

hd $x$ is the head (first element)

of the list $x$

$\operatorname{tl} x$ is the tail (list of remaining elements)

of the list $x$

cns $y x$ is the list with head $y$ and tail $x$

$\mathbf{u} x$ is the unit list with element $x$

Figure 9: Pairing and list operators.

$$
\begin{aligned}
l_{1} & =\lambda f \lambda a \lambda b \lambda x . f(a x) b \\
r_{1} & =\lambda f \lambda a \lambda b \lambda x . f a(b x) \\
l_{2} & =\lambda f \lambda a \lambda b . \mathbf{c n s}(\mathbf{h d} a)(f(\mathbf{t l} a) b) \\
r_{2} & =\lambda f \lambda a \lambda b . \mathbf{c n s}(\mathbf{h d} b)(f a(\mathbf{t} b)) \\
r & =\lambda o \cdot\left(r_{1}(\mathbf{f s t} o)\right)\left(r_{2}(\mathbf{s n d} o)\right) \\
l & =\lambda o \cdot\left(1_{1}(\mathbf{f s t} o)\right)\left(1_{2}(\mathbf{s n d} o)\right) \\
c & =\lambda o \cdot \lambda a \cdot \lambda b \cdot \mathbf{c n s}((\mathbf{f s t} o)(\mathbf{h d} a)(\mathbf{h d} b))((\mathbf{s n d} o)(\mathbf{t l} a)(\mathbf{t l} b))
\end{aligned}
$$

Figure 10: Operators projecting structured meanings. ro applies the semantic operation $o$, while projecting an additional focus from the second (right) argument. $l o$ applies the semantic operation $o$, while projecting an additional focus from the first (left) argument.

Operators such as $\mathbf{a}, l \mathbf{a}, r(l \mathbf{a})$, and $\mathbf{b}$ (which is the type-raised backward function application operator) have the type of ordered pairs. $l$ is defined in terms of $l_{1}$ and $l_{2}$ to map an ordered pair of this kind to another ordered pair. $c$ acts as an application operator which combines an operator like $r(l(\mathbf{a})$ with the two structured meanings denoted by the child phrases.

The operators $l, r$ and $c$ are defined in Figure 10, using the list operations of Figure 9. Figure 11 defines type-raised function application operators. It remains to define the focusing operator. As already exemplified above, this has a simple definition which adds the identify function to the front of the list denoted by the argument. This operation is defined in (27).

$$
\mathbf{f}=\lambda y \cdot \operatorname{cns}(\lambda x . x) y
$$

In sum, the l's and $r$ 's in representations such as Figure 6 are given a semantic interpretation as modifiers of semantic operations. When $l$ modifies a given opera- 


$$
\begin{aligned}
\text { ar } & =\lambda a \lambda b \cdot a b \\
\text { al } & =\lambda a \lambda b \cdot b a \\
\mathbf{f n l} & =\lambda a \lambda b \cdot \mathbf{n i l} \\
\mathbf{a} & =\text { pr ar fnl } \\
\mathbf{b} & =\text { pr al fnl }
\end{aligned}
$$

Figure 11: $\mathbf{a}$ is the type-raised rightward function application operator. $\mathbf{b}$ is the type-raised leftward function application operator. $\mathbf{b}$ and $\mathbf{a}$ can be modified by $l$ and $r$, e.g. $r(l(\mathbf{a}))$.

tion $o$, the combination $l o$ projects an extra widest-scope focus from the left child. When $r$ modifies an operation $o$, the combination $r o$ projects an extra widest-scope focus from the right child. A composite operation $l(r(o))$ projects a widest-scope focus from the left child, and a narrower-scope focus from the right child.

There is another part of the semantic analysis which I will only sketch. The $l r$ operators have a systematic correspondence with focusing operators. For instance we would not want a phrase whose right child contains no $\mathrm{F}$ to be annotated with the semantic operator $c(r \mathbf{a})$, since $r$ projects a focus from the right child. This kind of issue comes up in a couple of places in variable-free and structured approaches to compositional semantics. Pieces of meaning of functional type have certain intended interpretations (with one argument position corresponding to a free pronoun, another argument position corresponding to a free trace, another corresponding to a focus, and another corresponding to an ordinary compositional argument). These interpretations constrain how pieces of meaning can combine, and what type-raising operators are inserted in semantic composition. One way of controlling such as system is to use an enriched system of semantic-type labels to regulate semantic composition. For instance, consider a type system where a VP has the type $e t$, and a subject NP has type $e$. Given the phrase order [NP VP], these are combined with a leftward function composition operator of type $e(e t) t$. Suppose an NP with a free focus of type $e$ has a type label $e \triangleright e$. This is not an approriate argument for a leftward function application operator of type $e(e t) t$. The mismatch is fixed with a type-raising operator $l$ with type $(e(e t) t)(e \triangleright e)(e t)(e \triangleright t)$, which modifies rightward function application to propagate a focus from the first (left) argument. The ultimate value type $(e \triangleright t)$ type represents a standard type $t$ with a free focus of type $e$. On this approach, regulating the distribution of $l$ 's and $r$ 's is reduced to checking type consistency.

This completes the semantics of $l r$ sequences. The phonology is stated in (28). The constrant says that a node labeled with sequence which starts with $l$ must have a strong left child, while a node labeled with a sequence that starts with $r$ must have a strong right child.

(28) Phonology of $l r$ labels. 
i. The left child of any node labeled $l \alpha$ is stronger than the right child.

ii. The right child of any node labeled $r \alpha$ is stronger than the left child.

\section{Conclusion}

(29) is the new representation of the SOF configuration. The operators $l$ and $r$ fix the scope of the F's, and have a phonological interpretation in terms of metrical prominence.

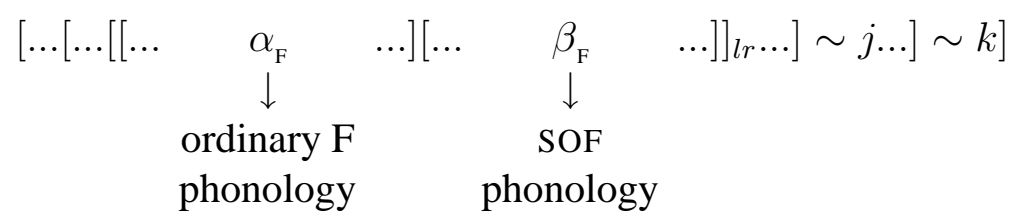

To complete the phonological part of the theory, the interpretation of the focusprojecting operators should be embedded in a account of prosodic phonology, including matters such as pitch accent assignment and the correspondence among metrical structure, phrasing, and syntactic structure, such as the one discussed in Féry and Ishihara (this volume).

The semantic solution in Section 4 is of a technical character. While it can be used in conjunction with a constrained architecture for focus interpretation such as alternative semantics, because alternatives can be introduced the top level, the recursive operations do not employ alternative semantics. Structured meanings are a semantic device which have also been used for other semantic problems, such as de re interpretation (Cresswell and von Stechow, 1982) and the exceptional scope of indefinites (Abusch, 1994). Therefore they should be regarded as a general device for constrained non-compositionality. It is hard to dismiss the employment of structured meaning devices on general grounds, especially if they are used in the interpretation of universal features. If the semantics of the feature in question is universal, it only has to be stipulated once in linguistic theory, and applying an a priori criterion of simplicity might lead in the direction of an incorrect theory of universal grammar. Still, as a methodological strategy, it is seems reasonable to use structured meanings only as a last resort.

Once we see the technical solution from Section 4, the possibility suggests itself of trying to do the same thing in recursive alternative semantics (Hamblin, 1973; Rooth, 1985; Shan, 2004). Perhaps the semantics of SOF configurations could be expressed with a tuple of meanings, consisting of an ordinary denotation, an alternative set representing the widest scope focus, and an alternative set representing the narrower scope focus. This is reminiscent of Büring's architecture for topic interpretation (Büring, 1997), and of Wagner's attempt to recast it in terms of foci with nested scopes (Wagner, 2008). My hunches about whether this strategy could work are mixed. 


\section{References}

Abusch, D. (1994). The scope of indefinites. Natural Language Semantics.

Beaver, D. and Clark, B. (2003). Always and only: Why not all focus-sensitive operators are alike. Natural Language Semantics.

Beaver, D., Clark, B., Flemming, E., Jaeger, T. F., and Wolters, M. (2007). When semantics meets phonetics: Acoustical studies of second-occurrence focus. Language, 83(2):283-341.

Büring, D. (1997). The Meaning of Topic and Focus - The 59th Street Bridge Accent. Routledge, London. Revised version of Tübingen doctoral dissertation.

Büring, D. (2006). Been there, marked that - a tentative theory of second occurrence focus. Unpublished manuscript.

Cresswell, M. and von Stechow, A. (1982). De re belief generalized. Linguistics and Philosophy, 5:503-35.

Fery, C. and Samek-Lodovici, V. (2006). Focus projection and prosodic prominence in nested foci. Language, 82.1 .

Hamblin, C. (1973). Questions in Montague English. Foundations of Language, pages $41-53$.

Howell, J. (2007). Second occurrence focus and the acoustics of prominence. In WCCFL 26, pages 114-125. Cascadilla Press Proceedings Project.

Jackendoff, R. (1972). Semantic Interpretation in Generative Grammar. MIT Press, Cambridge, Massachusetts.

Jacobson, P. (1999). Towards a variable-free semantics. Linguistics and Philosophy, 22:117-184.

Krifka, M. (1991). A compositonal semantics for multiple focus constructions. In Jacobs, J., editor, Informationsstruktur und Grammatik (Sonderheft 4, Linguistische Berichte). Opladen.

Krifka, M. (2004). Focus and/or context: a second look at second occurrence focus. In Kamp, H. and Partee, B., editors, Context dependence in the analysis of linguistic meaning. Elsevier.

Liberman, M. and Prince, A. (1977). On stress and linguistic rhythm. Linguistic Inquiry, pages 249-336.

Partee, B. (1991). Topic, focus, and quantification. In SALT 1, pages 159-187. 
Paulson, L. (1996). ML for the Working Programmer. Cambridge University Press, Cambridge, 2 edition.

Prince, A. (1983). Relating to the grid. Linguistic Inquiry, pages 19-100.

Rooth, M. (1985). Association with Focus. PhD thesis, University of Massachusetts.

Rooth, M. (1992). A theory of focus interpretation. Natural Language Semantics, 1(1):75-116.

Rooth, M. (1993). A hybrid architecture for focus. Handout for talk given at the Amsterdam colloquium.

Rooth, M. (1996a). Focus. In Lappin, S., editor, Handbook of Contemporary Semantic Theory, pages 271-297. Blackwell, Oxford.

Rooth, M. (1996b). On the interface principles for intonational focus. In Proceedings of SALT VI. CLC Publications.

Rooth, M. (2004). Comments on krifka's paper. In Kamp, H. and Partee, B., editors, Context dependence in the analysis of linguistic meaning. Elsevier.

Schwarzschild, R. (1999). Givenness, avoid $\mathrm{f}$ and other constraints on the placement of focus. Natural Language Semantics, 7(2):141-177.

Schwarzschild, R. (2004). Focus interpretations: Comments on geurts and van der sandt. Theoretical Linguistics, 30(1):137-47.

Selkirk, E. (2006a). Bengali intonation revisited: An optimality theoretic analaysis in which focus stress prominence drives focus phrasing. In Lee, C.-M., Gordon, M., and Buering, D., editors, In Topic and Focus: A Cross-Linguistic Perspective, pages 217-246. Kluwer Academic Publishers, Dordrecht.

Selkirk, L. (2006b). Contrastive focus, givenness, and phrase stress. Unpublished manuscript.

Sestoft, P. (1996). Standard ML on the web server: Visualizing lambda calculus reduction.

Shan, C. (2004). Binding alongside hamblin alternatives calls for variable-free semantics. In Proceedings of SALT XIV. CLC Publications.

Tancredi, C. (2005). Association with focus and discourse licensing. Presented at National Institute of Informatics Workshop on Focus.

Truckenbrodt, H. (1995). Phonological phrases-their relation to syntax, focus, and prominence. $\mathrm{PhD}$ thesis, MIT. 
von Fintel, K. (1994). Restrictions on quantifier domains. PhD thesis, UMass.

von Stechow, A. (1985/89). Focusing and backgrounding operators. Technical Report 6, Fachgruppe Sprachwissenschaft, Universität Konstanz.

Wagner, M. (2006). Givenness and locality. In Proceedings of SALT XVI. CLC Publications.

Wagner, M. (2008). A compositional theory of contrastive topics. In Proceedings of NELS 28. GLSA.

Wold, D. (1996). Long distance selective binding: The case of focus. In Proceedings of SALT VI. CLC Publications. 